\title{
Influence of oxygen dissolution history on reconstruction behavior of a stepped metal surface
}

\author{
T.P. Pearl ${ }^{1}$, S.B. Darling, L. Niu ${ }^{2}$, D.D. Koleske ${ }^{3}$, D.J. Gaspar ${ }^{4}$, S.F. King ${ }^{5}$, \\ S.J. Sibener* \\ Department of Chemistry, The James Franck Institute, The University of Chicago, 5640 S. Ellis Ave., Chicago, IL 60637, USA
}

Received 20 March 2002; in final form 20 July 2002

\begin{abstract}
We have observed that the reconstruction dynamics for stepped $\mathrm{Ni}(977)$ are influenced by the oxygen dissolution history of the crystal. Using a complementary approach incorporating both real- and reciprocal-space techniques, it is found that the upper end of the thermal range over which this stepped metal surface transforms from single to double steps increases with selvedge or sub-surface oxygen concentration. These results enhance our understanding of how adsorbate dissolution, and hence oxygen exposure history, modify energetic pathways for metallic oxidation.

(c) 2002 Elsevier Science B.V. All rights reserved.
\end{abstract}

\section{Introduction}

Incorporation of oxygen into transition metals en route to metallic oxidation, especially nickel, has been heavily studied [1-13]. Extensive efforts have been undertaken toward developing a more complete description of the initial mechanistic stages in

\footnotetext{
${ }^{*}$ Corresponding author. Fax: +773-702-5863.

E-mail address: s-sibener@uchicago.edu (S.J. Sibener).

${ }^{1}$ Present address: The Pennsylvania State University, 152 Davey Laboratory, University Park, PA 16802-6300, USA.

${ }^{2}$ Present address: Applied Materials, Inc., 3050 Bowers Ave, Santa Clara, CA 95054-3299, USA.

${ }^{3}$ Present address: Sandia National Lab, New Mexico, P.O. Box 5800, Albuquerque, NM 87185-0601, USA.

${ }^{4}$ Present address: Pacific Northwest National Lab, P.O. Box 999/MS K8-93, Richland, WA 99352, USA.

${ }^{5}$ Present address: US EPA, 77 W Jackson Blvd, Chicago, IL 60604-3950, USA.
}

the growth of oxide layers. Interest in understanding the formation and properties of oxides ranges from metallic corrosion mitigation to employment of thin oxide films as catalysts or catalytic supports. For metals that oxidize, incorporation can proceed via one of two processes: when oxygen exposure sufficiently exceeds the limit to form a chemisorbed surface overlayer, or when the system is heated to the point where it is energetically favorable to absorb oxygen by incorporation into the underlying metal lattice. However, unlike analogous sub-surface hydrogen systems [14], not much is understood regarding the role of oxidation history or the presence of sub-surface oxygen in the selvedge region of a crystal in further oxygen dissolution.

Stepped metal surfaces have been observed to undergo structural phase transitions in the presence of oxygen, commonly a reversible doubling of step terrace widths and step heights [15-23]. The 
exact conditions with respect to temperature range and oxygen coverage for doubling vary significantly between substrates. Stepped metals prone to oxygen-driven doubling can be further categorized depending on whether they support sub-surface oxygen in the form of oxide or metastable species. Incorporation of oxygen in general has been linked to the reversibility of the doubling process for stepped systems of nickel and rhodium [19-21]. Even though they exhibit vastly different oxidation and sub-surface oxygen behavior, nickel and rhodium stepped surfaces are more similar than platinum and copper counterparts that have a much smaller sticking coefficient for oxygen and are more resistant to forming oxides. Structural phase sensitivity of these high index surfaces to adsorbates like oxygen makes them particularly useful for probing the factors that influence adsorption and absorption of oxygen.

Oxygen-induced reconstruction behavior of $\mathrm{Ni}(977)$ has been thoroughly investigated in our group using both real- and reciprocal-space techniques $[20,21,24,25]$. In the presence of a small amount of adsorbed oxygen, less than $2 \%$ of a monolayer, the surface doubles over a thermal range that is limited on the low end by thermally promoted mobility of atoms including step meandering and at the high end by dissolution of the step-edge adsorbed oxygen. At temperatures where oxygen dissolves, the surface re-singles indicating the need for oxygen to stabilize merged steps at these relatively elevated temperatures. In this Letter, we are highlighting the variance at elevated temperature where double steps become unstable with respect to reentrant singling. We believe that the temperature at which double steps become unstable due to oxygen dissolution can be understood in light of the previous dissolution history of oxygen into the crystal. We present a montage of data from experiments on two different $\mathrm{Ni}(977)$ crystals that involved the interaction with oxygen at elevated temperatures.

\section{Experimental details}

Experiments were performed in two different ultra-high vacuum chambers described elsewhere
[26,27]. Various surface sensitive techniques have been employed for the study of oxygen interacting with $\mathrm{Ni}(977)$ including helium atom scattering (HAS), low energy electron diffraction (LEED), Auger electron spectroscopy (AES), and scanning tunneling microscopy (STM). This kinkless vicinal is derived from a miscut of a $\mathrm{Ni}\left(\begin{array}{lll}1 & 1 & 1\end{array}\right)$ crystal in the $\left[\begin{array}{lll}2 & \overline{1} & \overline{1}\end{array}\right]$ direction resulting in steps composed of (1 111 ) terraces that are eight atomic rows wide and monatomically high $\left(\begin{array}{lll}1 & 0 & 0\end{array}\right)$ risers. Over the course of different experiments performed in our group, two different $\mathrm{Ni}(977)$ crystals have been employed. The first sample, which will be denoted as $\mathrm{Ni}(977)$-heavy, was used primarily for oxidation studies, i.e., intentional growth of $\mathrm{NiO}\left(\begin{array}{lll}1 & 1 & 1\end{array}\right)$ observed with HAS and LEED [28]. For context, this crystal was repeatedly exposed to substantial doses of $\mathrm{O}_{2}(\sim 20 \mathrm{~L}$ per experiment) over a span of two years. Reconstruction behavior of a second sample was initially examined using HAS (light oxidation) and then monitored with STM after the sample had been used for related oxidation experiments (moderate oxidation). These oxidation studies were completed over the course of one year and involved smaller oxygen exposures (step-edge titration) than those for $\mathrm{Ni}(977)$-heavy. Therefore, three oxygen dissolution histories were probed; these crystals will be referred to as $\mathrm{Ni}(977)$-light, $\mathrm{Ni}(977)$-moderate, and Ni(9 7 7)-heavy. Typically, these crystals were cleaned by repeated cycles of sputtering with $1 \mathrm{keV} \mathrm{Ar}{ }^{+}$ions followed by annealing above $1000 \mathrm{~K}$ until carbon and sulfur levels were below our AES detection limit. Surface crystallinity was confirmed by a sharp LEED pattern with well-defined splitting of the (1 111$)$ terrace spots (characteristic of the stepped surface), as well as with helium diffraction. STM data of this surface exhibit a low kink density and narrow terrace width distribution with step-edge coherence extending over hundreds of nanometers.

\section{Results and discussion}

HAS was used to track oxygen-induced structural evolution of the $\mathrm{Ni}(977)$-heavy surface from single steps to double steps, back to single steps, and eventually to oxide in the presence of oxygen 
at $500 \mathrm{~K}$ (Fig. 1). Scattering in this experiment was performed across the step edges along the $\left[\begin{array}{ll}2 & \overline{1}\end{array}\right]$ azimuth (downstairs direction), revealing information regarding terrace widths. The distance between the two diffraction peaks is inversely proportional to the average terrace width on the surface. At the inception of the experiment, the two peaks correspond to diffraction from single steps. The center peak that appears at approximately $0.12 \mathrm{~L} \mathrm{O}_{2}$ exposure signals diffraction from double steps. During the course of these experiments, doubling was found to proceed at temperatures as high as $550 \mathrm{~K}$ [28]. This temperature is remarkably higher than the temperatures associated with oxygen dissolution for other $\mathrm{O} / \mathrm{Ni}$ systems $[8,11,29]$. Resingling of this crystal was never observed. Measurements above $550 \mathrm{~K}$ were not carried out when these experiments were originally performed - this was not an issue during the original study and oxygen absorptions since that time preclude performing the measurement now. The absence of observed resingling introduces some uncertainty into the location of the upper edge of the doubling thermal window. However, following the trend of the extent of doubling ver-

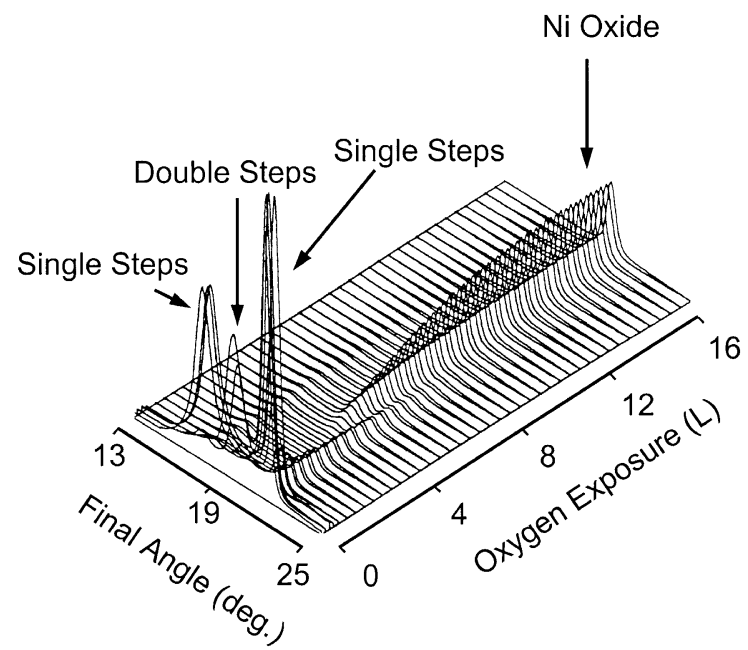

Fig. 1. Structural evolution of the Ni(977)-heavy surface while continuously dosing with $4 \times 10^{-9}$ Torr of oxygen at a crystal temperature of $500 \mathrm{~K}$. There are 40 sequential helium diffraction runs, with each run taking $40 \mathrm{~s}$. Scattering is across the steps in the 'downstairs' direction $\left(\theta_{\mathrm{i}}=30.4^{\circ}, E_{\mathrm{i}}=20.7 \mathrm{meV}\right)$ with $\theta_{i}$ measured from the terrace normal. sus temperature for $\mathrm{Ni}(977)$-heavy it is clear that resingling would not occur below $570 \mathrm{~K}$, if at all.

In order to study the reconstruction dynamics with HAS in the limit of small amounts of adsorbed oxygen, the second $\mathrm{Ni}(977)$ sample was acquired. Fig. 13 of [19] illustrates the sensitivity of the surface reconstruction to temperature. Plotted is the scattering intensity from double height steps as a function of time for a single dose of oxygen at different temperatures. As shown in Fig. 13 of [19], doubling does not proceed at $500 \mathrm{~K}$ and at $470 \mathrm{~K}$ double steps begin to grow in but the process then reverses and starts to re-single. The chemical potential of the selvedge region can be modified by the presence of oxygen, which in turn influences the thermodynamics of partitioning between surface adsorbed and sub-surface absorbed oxygen. Variations in oxygen segregation can account for changes in the stabilization regime of the double step phase. The nature of the dissolution history for $\mathrm{Ni}(977)$-heavy made it possible to actually have double step phase stability at higher temperatures compared to Ni(977)-light.

Exploration of the rich reconstruction behavior was further probed with LEED and STM after the Ni(977)-light sample had undergone oxidation experiments short of inducing irreversible faceting to produce $\mathrm{Ni}(977)$-moderate. In the course of mapping out the phase diagram for doubling and singling of the Ni(977)-moderate crystal, LEED revealed a significant temperature shift, from 475 to $570 \mathrm{~K}$, for re-singling of the surface [21]. This is the same trend in modification of the dynamic range for surface reconstruction observed between $\mathrm{Ni}(977)$-heavy and Ni(9 77 )-light. A related study on the behavior of the $p(2 \times 2)-\mathrm{O}$ system on this crystal showed that this temperature coincides with AES measurements of where oxygen completely dissolves [30]. Note, too, that dissolution temperature varies with oxidation history of the crystal for Ni(1 11 ) [29]. Fig. 2 summarizes the widening temperature windows following cumulative oxygen absorption; also shown are illustrative STM images of singled and doubled Ni(977) surfaces. Time-resolved STM imaging of the reconstruction revealed many of the local mechanistic steps involved in this step merging phenomenon [24,25]. Final state imaging was 


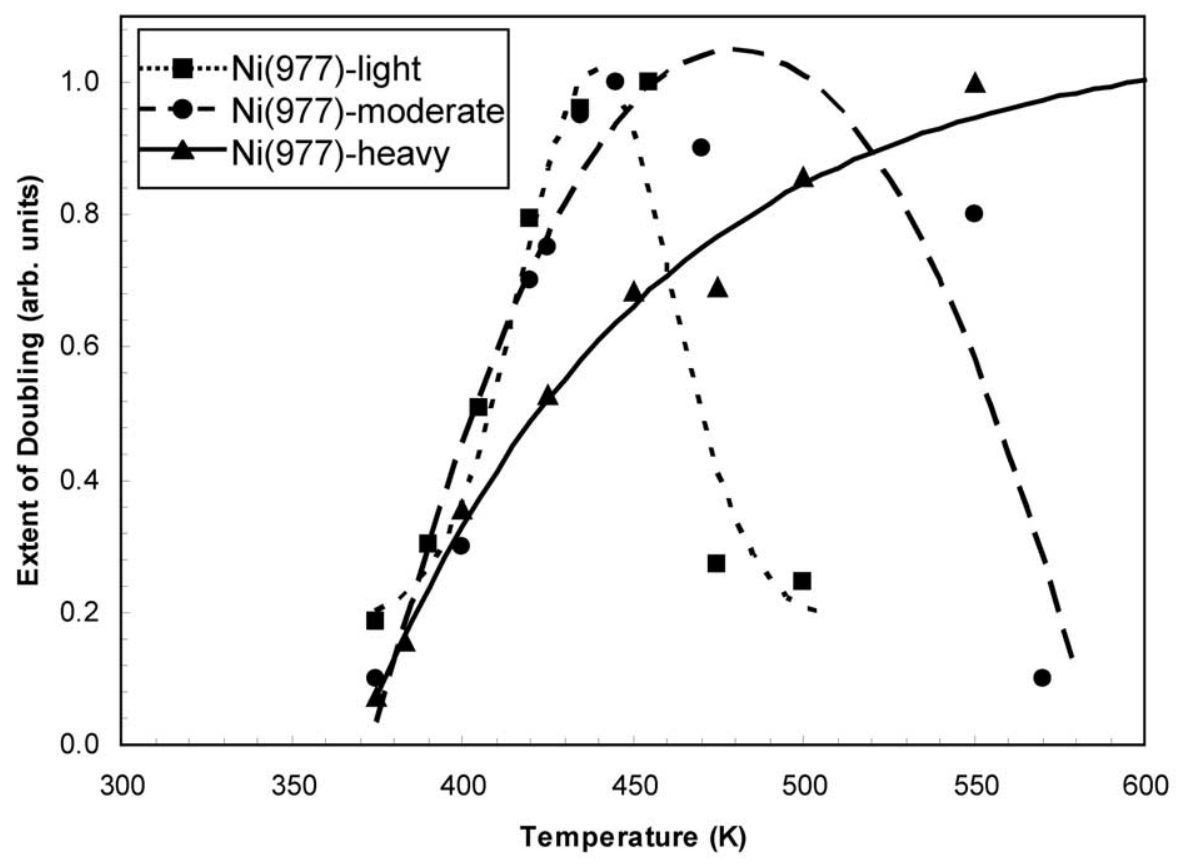

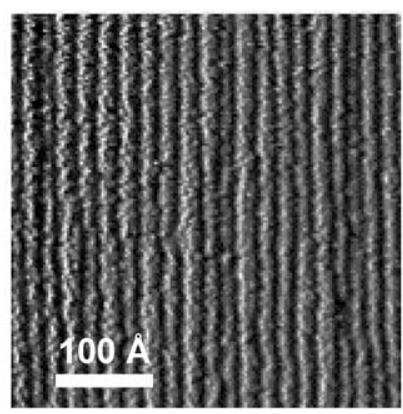

Initial Singled Structure

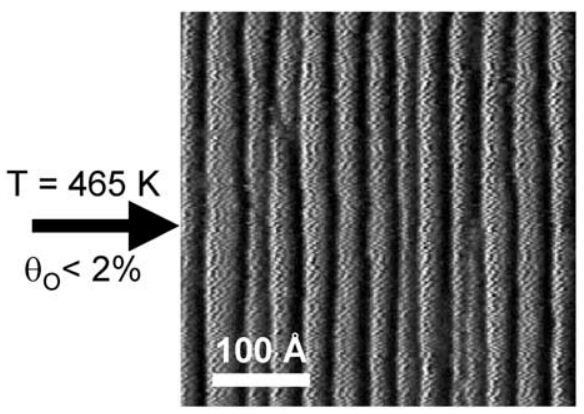

Doubled Structure

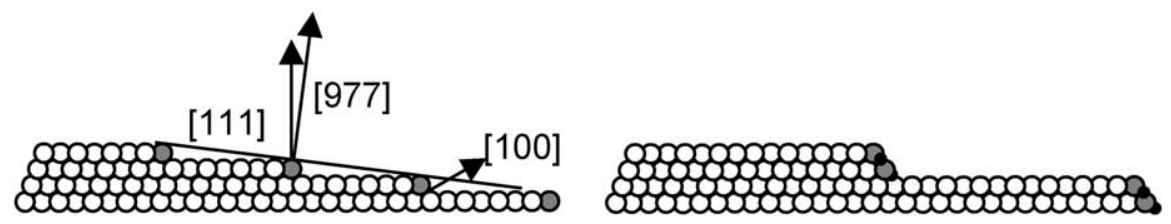

Fig. 2. Extent of $\mathrm{Ni}(977)$ doubling as a function of surface temperature for three different oxygen absorption histories. The initial transition from singled to doubled remains constant at $375 \mathrm{~K}$, but the temperature at which re-singling occurs increases monotonically with increased oxidation history: 470, 570, and $>570 \mathrm{~K}$ for a lightly, moderately, and heavily oxidized crystal, respectively. The extension beyond $550 \mathrm{~K}$ for the heavily oxidized crystal is an extrapolation based on the observed trend; measurements at temperatures higher than $550 \mathrm{~K}$ were not carried out as part of this study. The fit lines are included to visualize the systematic trend in the three data sets. STM parameters for the illustrative images: $400 \times 400 \AA, 100 \mathrm{mV}$ positive sample bias with respect to the tip, $1 \mathrm{nA}$ tunneling current. Side view schematic drawings of the single and double step phases are also shown below their corresponding STM images. 
performed after the surface reconstruction was driven to completion in order to corroborate LEED observations of doubling occurring at higher temperatures for the $\mathrm{Ni}(977)$-moderate crystal than for Ni(977)-light. STM sequences for temperatures as high as $470 \mathrm{~K}$ were acquired which showed no indication of a kinetic competition as previously observed with HAS in the earlier experiment. The large shift in the temperature associated with re-singling of the $\mathrm{Ni}(977)$-moderate sample is attributed to cumulative absorption of oxygen from previous experiments including oxidation experiments performed after the low coverages studies using $\mathrm{Ni}(977)$-light were completed. While the sub-surface concentration of oxygen was not measured for the crystals studied, the observed shifts in reconstruction dynamics are monotonically increasing as a function of integrated oxygen exposure and are concomitant with our understanding of the strong effect oxygen plays as both an adsorbate and an absorbate in influencing surface energetics. Quantitative assessment of the extent of oxygen dissolution would in fact be difficult and would require the ability to distinguish between oxygen that populates the first few layers in the sub-surface and oxygen that is adsorbed on the outer surface only.

Various experiments in our group have been aimed at understanding the role of steps in stabilizing chemisorbed overlayers [30-32]. Oxygen preferentially adsorbs at step edges [23,33] and dissolves at higher temperatures than oxygen located on the terraces of the stepped surface [30]. These data show that the dissolution temperature for the step-edge adsorbed oxygen has been modified by the previous oxidation and oxygen incorporation. In the previous experiment using HAS on $\mathrm{Ni}(977)$-light, the dissolution temperature was lower than $500 \mathrm{~K}$.

A study of oxygen dissolution rates for $\mathrm{Ni}\left(\begin{array}{lll}1 & 1 & 0\end{array}\right)$ by Holloway and Outlaw [8] revealed that rates of absorption can also be influenced by surface phase stability as opposed to only kinetic limitations. They observed the dissolution temperature for chemisorbed oxygen steadily increase by as much as $100 \mathrm{~K}$ following successive absorptions of oxygen. However, when they repeated the experiment and sputter cleaned the surface between oxygen exposures, the temperature at which oxygen was dissolved remained unchanged. This result cleanly demonstrated that oxygen absorption history can significantly affect subsequent surface physics. Using the remarkable sensitivity of the $\mathrm{Ni}(977)$ reconstruction to oxygen partitioning we have been able to observe related behavior to that seen by Holloway and Outlaw with our results clearly illustrating structural and chemical ramifications for the material.

\section{Summary}

We have shown that the thermal range over which the doubling occurs can be extended as the oxygen concentration in the crystal selvedge region increases with multiple dissolutions. Dissolution history directly influences structural phase stability by altering the driving forces governing selvedge and bulk oxygen absorption. Interestingly, the modifications in dissolution thermodynamics and kinetics are irreversible. Oxygen absorption into the stepped crystals has been modified permanently even though the crystal structure has not been altered by faceting. Cross-talk between bulk, i.e., selvedge, and surface oxygen is remarkably influential in determining phase stability with ramifications extending to equilibrium morphology and rates of metallic oxidation.

\section{Acknowledgements}

This work was supported by the Multidisciplinary University Research Initiative of the Air Force Office of Scientific Research.

\section{References}

[1] M.J. Stirniman, W. Li, S.J. Sibener, J. Vac. Sci. Technol. A 13 (1995) 1574.

[2] W. Li, M.J. Stirniman, S.J. Sibener, Surf. Sci. 329 (1995) L593.

[3] B.D. Zion, A.T. Hanbicki, S.J. Sibener, Surf. Sci. 417 (1998) L1154.

[4] S.A. Raspopov, A.G. Gusakov, A.G. Voropayev, A.A. Vecher, B.K. Grishin, J. Alloy Compd. 227 (1995) 5. 
[5] W.-D. Wang, N.J. Wu, P.A. Thiel, J. Chem. Phys. 92 (1990) 2025.

[6] P.H. Holloway, J.B. Hudson, Surf. Sci. 43 (1974) 123.

[7] P.H. Holloway, J.B. Hudson, Surf. Sci. 43 (1974) 141.

[8] P.H. Holloway, J. Vac. Sci. Technol. 18 (1981) 653.

[9] A. Atkinson, D.W. Smart, J. Electrochem. Soc. 135 (1988) 2886.

[10] O.L. Warren, P.A. Thiel, J. Chem. Phys. 100 (1994) 659.

[11] C.R. Brundle, J.Q. Broughton, in: A. King, D.P. Woodruff (Eds.), The Chemical Physics of Solid Surfaces and Heterogeneous Catalysis, Elsevier, Amsterdam, 1990, p. 132.

[12] B. Chakraborty, S. Holloway, J.K. Nørskov, Surf. Sci. 152/ 153 (1985) 660.

[13] P. Nordlander, M. Ronay, Phys. Rev. B 36 (1987) 4982.

[14] S.T. Ceyer, Acc. Chem. Res. 34 (2001) 737.

[15] J.M. Blakely, R.L. Schwoebel, Surf. Sci. 26 (1971) 321.

[16] D.W. Blakely, G.A. Somorjai, Surf. Sci. 65 (1977) 419.

[17] D.G. Castner, G.A. Somorjai, Surf. Sci. 83 (1979) 60.

[18] G. Comsa, G. Mechtersheimer, B. Poelsema, Surf. Sci. 119 (1982) 159.

[19] G. Hoogers, D.A. King, Surf. Sci. 286 (1993) 306.
[20] L. Niu, D.D. Koleske, D.J. Gaspar, S.F. King, S.J. Sibener, Surf. Sci. 356 (1996) 144.

[21] T.P. Pearl, S.J. Sibener, J. Chem. Phys. 115 (2001) 1916.

[22] G.A. Somorjai, Chemistry in Two Dimensions: Surfaces, Cornell University Press, Ithaca, 1971.

[23] G. Witte, J. Braun, D. Nowack, L. Bartels, B. Neu, G. Meyer, Phys. Rev. B 58 (1998) 13224.

[24] T.P. Pearl, S.J. Sibener, J. Phys. Chem. B 105 (2001) 6300.

[25] T.P. Pearl, S.J. Sibener, Surf. Sci. Lett. 496 (2002) L29.

[26] B. Gans, S.F. King, P.A. Knipp, D.D. Koleske, S.J. Sibener, Surf. Sci. 264 (1992) 81.

[27] T.P. Pearl, S.J. Sibener, Rev. Sci. Instrum. 71 (2000) 124.

[28] D.D. Koleske, Ph.D. Thesis, University of Chicago, Chicago, 1992.

[29] A.R. Kortan, R.L. Park, Phys. Rev. B 23 (1981) 6340.

[30] T.P. Pearl, S.B. Darling, S.J. Sibener, Surf. Sci. 491 (2001) 140.

[31] A.T. Hanbicki, S.B. Darling, D.J. Gaspar, S.J. Sibener, J. Chem. Phys. 111 (1999) 9053.

[32] S.B. Darling, A.W. Rosenbaum, S.J. Sibener, Surf. Sci. 478 (2001) L313.

[33] K.A. Thompson, C.S. Fadley, Surf. Sci. 146 (1984) 281. 